\title{
On the reliability of the fractal dimension measure of solar magnetic features and on its variation with solar activity ${ }^{\star}$
}

\author{
S. Criscuoli ${ }^{1,2}$, M. P. Rast ${ }^{3}$, I. Ermolli ${ }^{4}$, and M. Centrone ${ }^{4}$ \\ 1 Dipartimento di Fisica, Università di Roma "Tor Vergata", via della Ricerca Scientifica 1, 00133 Roma, Italia \\ 2 High Altitude Observatory, NCAR, Boulder, CO 80307-3000, USA \\ e-mail: serenac@hao.ucar.edu \\ ${ }^{3}$ Laboratory for Atmospheric and Space Physics, Department of Astrophysical and Planetary Sciences, University of Colorado, \\ Boulder CO 80309, USA \\ 4 INAF - Osservatorio astronomico di Roma, via Frascati 33, 00040 Monte Porzio Catone, Italia
}

Received 30 June 2006 / Accepted 15 September 2006

\section{ABSTRACT}

\begin{abstract}
Context. Several studies have investigated the fractal and multifractal nature of magnetic features in the solar photosphere and its variation with the solar magnetic activity cycle.

Aims. Here we extend those studies by examining the fractal geometry of bright magnetic features at higher atmospheric levels, specifically in the solar chromosphere. We analyze structures identified in CaIIK images obtained with the Precision Solar Photometric Telescopes (PSPTs) at Osservatorio Astronomico di Roma (OAR) and Mauna Loa Solar Observatory (MLSO).

Methods. Fractal dimension estimates depend on the estimator employed, the quality of the images, and the structure identification techniques used. We examine both real and simulated data and employ two different perimeter-area estimators in order to understand the sensitivity of the deduced fractal properties to pixelization and image quality.

Results. The fractal dimension of bright "magnetic" features in CaIIK images ranges between values of 1.2 and 1.7 for small and large structures respectively. This size dependency largely reflects the importance of image pixelization in the measurement of small objects. The fractal dimension of chromospheric features does not show any clear systematic variation with time over the period examined, the descending phase of solar cycle 23.

Conclusions. These conclusions, and the analysis of both real and synthetic images on which they are based, are important in the interpretation of previously reported results.
\end{abstract}

Key words. Sun: activity - Sun: chromosphere - Sun: magnetic fields

\section{Introduction}

The spatial distribution of the solar magnetic fields is very complex, depending on both the varying level of magnetic activity over the solar cycle and the height of observation in the solar atmosphere. This complexity likely results from both the dynamo process itself, which may occur on many different spatial scales, and the interaction of the field with convective motions as it emerges through the Sun's outer layers. The signatures of these processes have been investigated in previous works by fractal analyses of solar active regions, but the quantitative results obtained differ widely depending on the type of data and analysis techniques employed (e.g. Janssen et al. 2003; McAteer et al. 2005). Moreover, the lack of a unique definition of the fractal dimension itself often makes comparison of results difficult.

Among recent studies, one focused on the possible relationship between magnetic feature complexity and solar cycle phase (Meunier 2004). This study looked at a large sample of active region magnetograms acquired with the Michelson Doppler Interferometer (MDI) aboard the Solar and Heliospheric Observatory (SOHO) between April 1996 and June 2002. The measured fractal dimension increased with structure size (in agreement with Meunier 1999; and

$\star$ Appendix A is only available in electronic form at http://www . aanda.org
Nesme-Ribes et al. 1996), showing a peculiar change in behavior near structures of area 550-800 $\mathrm{Mm}^{2}$. A similar dependence on structure size was also found by Janssen et al. (2003) in both high resolution photospheric magnetograms and magnetohydrodynamic (MHD) simulations. Additionally, Meunier (2004) investigated the relationship between the geometry of facular structures of different spatial scales and magnetic field intensity, flare activity, and solar cycle phase. She found that, while a region complexity generally increases with magnetic field intensity, there is no clear correlation with flare activity. Variations of fractal dimension with solar cycle were also reported, but their amplitudes and sign largely depended on object size and the associated magnetic field.

In order to investigate the complexity of magnetic features using observations representative of chromospheric heights, we have analyzed the fractal dimension of bright features identified in full-disk CaIIK images acquired by the Precision Solar Photometric Telescopes (PSPTs) at Osservatorio Astronomico di Roma (OAR) and Mauna Loa Solar Observatory (MLSO). The data analyzed span the past 6 years and thus allow investigations of variation with the solar cycle.

Several factors can influence fractal dimension estimation. Both image resolution and projection effect correlation, mass function, and perimeter-area estimators in studies of interstellar molecular clouds (Sànchez et al. 2005; Vogelaar \& Wakker 1994), and resolution and thresholding effects are important in 
fractal dimension estimation of flow patterns in field soil by boxcounting methods (Baveye et al. 1998). Lawrence et al. (1996) studied similar effects in multifractal and fractal measures (boxcounting, cluster dimension, threshold set) of solar magnetic active regions. The effect of structure selection technique was also investigated by Meunier (1999, 2004).

To link our findings directly to those of several recent solar studies (for example Meunier 2004; Janssen et al. 2003) we have employed the perimeter-area relationship to define the fractal dimension of the identified features. To interpret our results, we have investigate the sensitivity of the deduced fractal dimension to the pixelization and resolution of the image and to the perimeter measure algorithm employed. In particular, we have determined how these factors influence the geometric properties deduced for objects of different sizes, and more generally have addressed the question of whether the perimeter-area relation is suitable to the study of the fractal and multifractal nature of solar magnetic features as a function of the solar cycle.

This paper is organized as follows. In the next section we describe the observations, data processing techniques, and geometrical measures employed. In Sect. 3 we present the results obtained and in Sect. 4 compare them to those of previous efforts. In Sect. 5 we investigate the sensitivity of the deduced fractal dimension to the image resolution and the measurement techniques employed, by examining synthetic structures whose fractal properties are theoretically known: non-fractal objects, von Koch snowflakes, and those produced by fractional Brownian motion (fBm). The effect of atmospheric seeing is also investigated using PSPT images taken under variable seeing conditions. In Sect. 6 we discuss our and previous results, in light of the conclusions drawn in Sect. 5. Finally, in Sect. 7 we summarize our work and conclude with a more general discussion on the validity of the methods.

\section{Observations, processing and definitions}

\subsection{PSPT data}

The bulk of the data we analyzed is from the archive of daily full-disk observations carried out with the PSPT at OAR. This was supplemented with data from the PSPT at MLSO for consistency and resolution tests (Sects. 3.1 and 5.2). Details about the data and the image pre-processing can be found in Ermolli et al. (2006). In brief, the images were taken with "twin" telescopes at the two sites, through interference filters centered at three wavelength bands (CaIIK line center $393.4 \mathrm{~nm}, F W H M 0.27 \mathrm{~nm}$, blue continuum $409.4 \mathrm{~nm}, F W H M 0.27 \mathrm{~nm}$, and red continuum $607.1 \mathrm{~nm}, F W H M 0.46 \mathrm{~nm}$ ), with a $2048 \times 204816 \mathrm{bit} /$ pixel CCD camera, yielding a spatial scale of $\sim 1^{\prime \prime}$ per pixel. Images from OAR were binned to half resolution, yielding a final spatial scale of $\sim 2^{\prime \prime}$ per pixel. Images from the two telescopes were independently dark and flat-field corrected and had the mean center-to-limb variation removed. The images of any one wavelength triplet were resized and aligned to allow pixel by pixel comparison between filters.

For this study we selected OAR daily image triplets, obtained on 238 different observing days during the summers (July to September) of 2000 through 2005 . We chose images acquired during the summer months because these are generally of higher quality. In order to compare results obtained with the two instruments, we also selected 44 triplets (the best in the CaIIK band of the day, according to quality criteria described in Sect. 2.2) from the MLSO and OAR archive taken during the summer of 2005. For that comparison, MLSO images were rescaled to match OAR spatial scale images ( $2^{\prime \prime}$ per pixel).

Finally we were able to quantify the effects of atmospheric seeing by using MLSO images acquired at $10 \mathrm{~min}$ intervals throughout the day, weather permitting. According to the quality criteria explained in next paragraph, we selected 27 pairs of high and low quality triplets (one pair per day) from the period February to October 2005. For this analyses, the full resolution $\left(\sim 1^{\prime \prime}\right.$ per pixel) MLSO data were employed.

\subsection{Data quality}

The geometric properties of solar features extracted from the images are likely sensitive to the spatial resolution of the image being analyzed. This in turn depends on atmospheric and instrumental operation conditions during the observation. To estimate the inherent quality of any given data image, we measured (in pixel units) the width of a Gaussian fit to the limb profile observed in CaIIK images. Small values of the solar limb width indicate lower instrumental or atmospheric smearing and thus better quality images. The limb width distributions of our datasets are asymmetrically shaped with a long tail toward higher values, so that the mean is not the most probable value. The mean limb profile width of the OAR CaIIK (binned to half resolution) images analyzed is $2.5 \pm 4.0$ pixels with a median value of 1.4 pixels. That of the MLSO CaIIK images from the summer 2005 is $4.1 \pm 0.8$ pixels for the mean and 3.9 pixels for the median (measured on full resolution images), while that for the OAR images acquired in the same period are $4.0 \pm 6.5$ pixels and 2.2 pixels respectively (measured on binned half resolution images). Considering the different pixel scale of MLSO and OAR images, the two 2005 datasets have similar median quality (about 4 arcsec), but the OAR dataset contains a higher number of low quality images, skewing the mean to a much higher value.

The MLSO limb width distribution for year 2005 has its maximum at a value of about 3.7 pixels (measure on full resolution images). To study fractal dimension dependence on seeing condition (Sect. 5.2), images from each day were grouped in two sets: those whose limb widths lie below $0.1 \sigma$ from the peak, and those whose limb widths lie between $0.8 \sigma$ and $3.0 \sigma$ above the peak. From each of these groups, the best and the worst images were selected, so that for each observing day, two different quality images were retained. This restricted our analysis of seeing effects to 27 observing days. The mean limb width for the two groups were $3.5 \pm 0.2$ and $4.9 \pm 0.3$ for high and low quality images respectively.

\subsection{Feature identification}

Bright features were identified in the CaIIK images using two methods based on a combination of pixel intensity and connectivity. Because of the link between CaIIK brightness and magnetic flux intensity (e.g. Skumanich et al. 1975; Harvey \& White 1999; Rast 2003; Ortiz \& Rast 2005) these features are assumed to represent small magnetic structures, but this assumption plays no role in their identification.

The first identification method is analogous to that used by Meunier (2004), taking into account intensity alone and based on fixed thresholding values. While Meunier (2004) employed thresholding on magnetograms, we select pixels whose intensity 

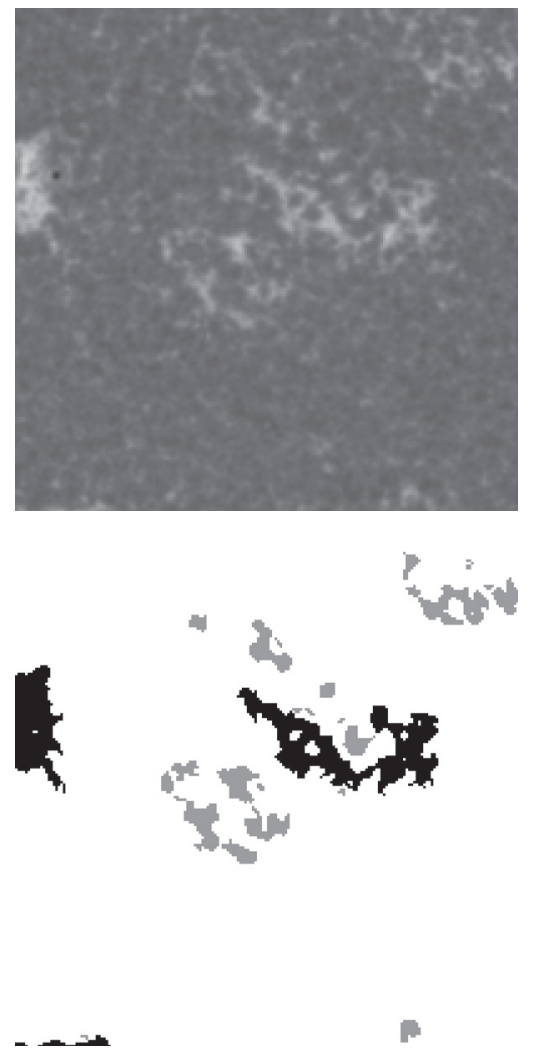

Fig. 1. Central disk detail of a CaIIK image from OAR PSPT archive and corresponding mask obtained with the second identification method described in the text. Features with areas lower and larger than $2000 \mathrm{Mm}^{2}$ are shown in gray and black colors respectively.

contrast $^{1}$ in the CaIIK images exceeds a given value. Umbral, penumbral and pore pixels, were included selecting those pixels whose intensity in red continuum images was below a given threshold.

The second identification method applied takes into account both pixel intensity and connectivity. As described in detail by Ermolli et al. (2006), we first identified regions of the solar disk which include active regions and their remnants. These preselected regions are made up of those pixels which are brighter than a fixed contrast value on a spatially filtered image (box average side $\left.=\frac{R_{\text {sun }}}{20}\right)$. A second contrast threshold value, determined by the procedure explained in Nesme-Ribes et al. (1996), is then used to single out from the pre-selected regions all those features identified for our study. Pixels representative of sunspots and pores, previously identified from red continuum images, were excluded from the identified features.

For the subsequent fractal analysis, the two identification methods were employed to produced two independent binary masks from each image triplet processed. In each of these pixels satisfying one of the two identification criteria above were assigned a value of one, with all other pixels set to zero. An example of a CaIIK PSPT image (showing only a central disk region) and the corresponding mask, obtained with the second identification method described, is given in Fig. 1. To reduce distortion due to projection effects, the analysis was restricted to structures near disk center, $\mu>0.8$, where $\mu$ is the cosine of the heliocentric position angle. Additionally, isolated bright points were

${ }^{1} I_{\mathrm{c}}=\frac{I-I_{0}}{I_{0}}$, where $I$ is the intensity measured at each pixel and $I_{0}$ is that representative of the quiet sun and obtained by a fit to its center to limb intensity variation. removed from consideration by discarding all structures of area less than 10 pixel $^{2}$.

\subsection{Perimeter and area evaluation}

There are several ways to define and evaluate the perimeters and areas of features in a binary image (Gonzalez \& Woods 2002), and thus characterize the independent structures. The goal is to define, detect, and count the pixels which constitute the feature edges. For our study we considered three methods, and evaluated the errors associated with them.

In the first method, we defined border pixels by row and column, identifying for each the pixel for which the binary value changes. The perimeter was then evaluated by summing the external sides of the border pixels, so that for example an object made up of 1 pixel has an area of 1 and a perimeter of 4 , while one made up of two pixels has an area of 2 and a perimeter of 6 or 8 depending on the pixels' relative positions.

In the second method, we applied the Roberts operator (Turner et al. 1998) to the image in order to identify border pixels and defined the perimeter as the sum of the all pixels whose value is not zero. Using this method, an object of 1 pixel has a perimeter of 4 and an object of two pixels always has a perimeter 6 , independent of the relative positions.

In the third method, pixels are identified as border pixels if they are connected from between 1 and 7 of the neighboring 8 contiguous pixels. The perimeter is the sum of the selected pixels, so that an object 1 pixel in area has a perimeter of 1 and an object of area 2 has a perimeter of 2 , independent of the pixels' relative positions.

Only the results obtained using the first method are included in the body of this paper. The reasons for this are discussed in Sect. 5.1.

\subsection{Fractal dimension definition}

Several definitions of the fractal dimension of two-dimensional structures and corresponding techniques for its estimation exist (Turner et al. 1998).

If a structure is self-similar, its perimeter $L$ and area $A$ display a power-law relation:

$L \propto A^{d / 2}$

where $d$ is the fractal dimension. With this definition, $1 \leq d \leq 2$ and $d=1$ for non-fractal structures.

We estimated $d$ using two methods. In the first, we performed a simple linear fit to the logarithm of the perimeters and areas measured for structures of different sizes; we indicate the fractal dimension so obtained as $D$. In order to investigate the size dependence of the fractal dimension estimated in this way, the fit is performed over the entire data set or over objects in a specified size range. In the second, we adopted a method first proposed by Nesme-Ribes et al. (1996) and later employed by Meunier (1999, 2004), in which perimeter and area values are averaged over bins in area, each of width $\Delta \log A=0.05$, and the fitting is done on these averages for a series of overlapping windows of constant width $\Delta \log A=1.5$, producing a measure of $d$ which is a function of $A$. We indicate the fractal dimension estimated in this way as $d 1$. For both methods linear fits were performed by a chi square minimization and the associated error is taken to be the variance in the estimate of the slope.

More details about the significance of the two estimators and how they relate to each other are given in the Appendix. 


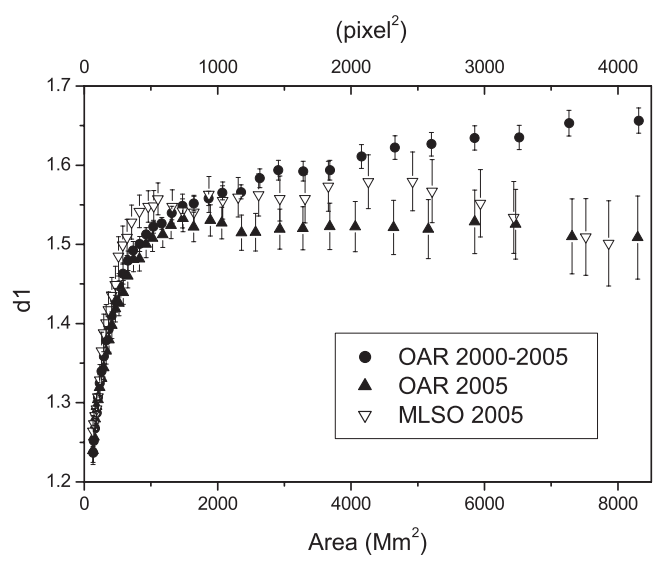

Fig. 2. Fractal dimension $d 1$ versus area of bright features identified on calcium images ( $2^{\prime \prime} /$ pixel). Full circles: summers $2000-2005$ OARPSPT. Open triangles: summer 2005 MLSO-PSPT. Full triangles: summer 2005 OAR-PSPT. $d 1$ increases fast with object size at area smaller than $2000 \mathrm{Mm}^{2}$. For larger areas, a plateau is observed for summer 2005 OAR and MLSO data, and a slow rise on the 2000-2005 OAR dataset.

Table 1. Fractal dimension $D$ estimated for features selected on OARPSPT and MLSO-PSPT CAIIK images. First row: fractal dimension $D$ considering the entire range of structure areas. Second row: fractal dimension $D$ considering only structures larger than $2000 \mathrm{Mm}^{2}$.

\begin{tabular}{ccc}
\hline \hline OAR 2000-2005 & OAR 2005 & MLSO 2005 \\
\hline $1.337 \pm 0.002$ & $1.307 \pm 0.003$ & $1.307 \pm 0.004$ \\
$1.64 \pm 0.02$ & $1.53 \pm 0.07$ & $1.54 \pm 0.09$ \\
\hline
\end{tabular}

\section{Results}

\subsection{Fractal dimension and feature size}

Figure 2 shows the variation in fractal dimension $d 1$ of the identified chromospheric features as a function of their size, as derived from the OAR and MLSO PSPT CaIIK rebinned data using the second identification method described in Sect. 2.3. The results obtained from three data sets are shown: the full 2000-2005 OAR summer period, the single 2005 summer OAR data, and the single summer 2005 MLSO data. For all data sets, $d 1$ increases with object size, increasing fastest for structures of smallest areas and becoming almost constant at the largest scales. The three curves overlap for structures of area less than about $1000 \mathrm{Mm}^{2}$, corresponding to about 500 pixel $^{2}$. For objects of size greater than about $1000-1500 \mathrm{Mm}^{2}$ the 2005 OAR and MLSO data both show a plateau in the measured fractal dimension. Somewhat surprisingly, this plateau is less evident when analyzing structures from the full 2000-2005 OAR data set. The fractal dimension deduced over this longer period continues to slowly increase even at the largest scales.

Table 1 shows the value of $D$ obtained from the different data sets when a single fit to the perimeter area relation is made over structures of all sizes (top row) or only those of area larger than $2000 \mathrm{Mm}^{2}$ (bottom row), the threshold value suggested by the trends observed in Fig. 2. In agreement with $d 1$ estimates, the fractal dimension $D$ is reduced by the inclusion of the small and apparently less complex regions. In this case, because of the large number of objects with small areas, $D$ is biased toward a low value and the formal error quoted is quite small (as is a $\chi^{2}$ measure of the fit) in spite of the fact that a single linear fit does not reflect the perimeter area relation at all scales (see also Appendix). The large objects, who's perimeter area relationship is poorly fit by the single slope estimator, are

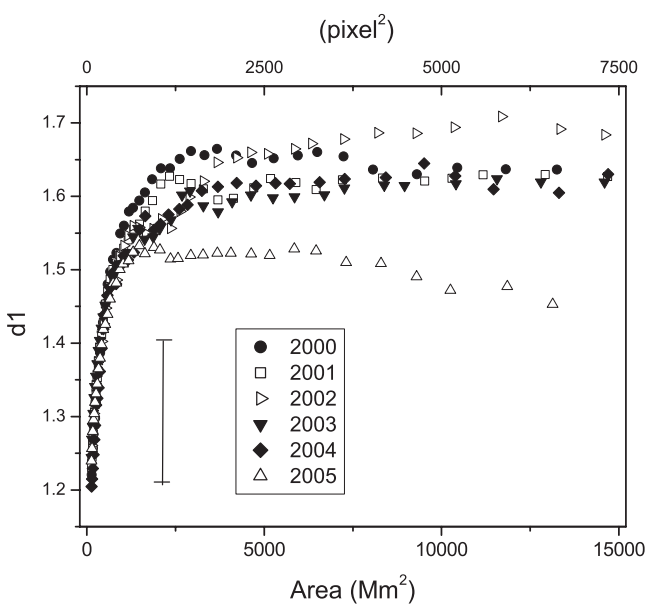

Fig. 3. Temporal variation of the fractal dimension $d 1$ versus area for features identified on OAR-PSPT calcium images. The bar on the left represents the largest error bar, obtained for the largest areas for year 2004. At area smaller than about $1000 \mathrm{Mm}^{2}$ all the curves overlap, while differences (not clearly correlated with solar cycle) are observed at the largest areas.

insufficient in number to significantly alter the fit value or influence the error measure. Figure 1 displays typically structures of areas larger and smaller than $2000 \mathrm{Mm}^{2}$. The smallest objects appear somewhat rounder and more regular than the largest ones, but no overwhelming difference between the two groups can be inferred by visual inspection alone.

\subsection{Temporal variation}

Figure 3 shows the variation of fractal dimension $d 1$ with both feature size and time for the six year OAR period analyzed. For sake of clarity, only the largest error bar (belonging to the largest area objects of year 2004) is indicated on the plot. The fluctuations in the other values shown are generally smaller than the largest differences observed among the years. We find that the variation in fractal dimension does not show a clear correlation with solar cycle over the period analyzed (the descending phase of Solar Cycle 23). The values for large structures show significant year to year variation, with the maximum and the minimum dimensions measured for years 2002 and 2005 respectively. The reliability of the 2005 values are supported by the nearly identical results obtained from the independent OAR and MLSO measurements (Table 1, Fig. 2). The other years show a plateau value at about 1.6 , although in the area range $2500-7000 \mathrm{Mm}^{2}$ year 2000 has a mean value of about 1.65 and a slight increase with object size up to a maximum value of 1.7 is measured for year 2002. Note that if we restrict the analyses to the area range $2500-7000 \mathrm{Mm}^{2}$ then a weak trend with the last cycle, showing a double activity peak in 2000 and 2002, is observed. Figure 4 shows the temporal variation in $D$ for three different area ranges. For the reasons explained in Sect. 3.1, error is omitted when fit is performed on the entire area range and at the smallest areas. In this measure a small trend with solar cycle is observed when the entire structure size range is included in the fit. When fit is performed on the largest objects, the highest fractal dimension is measured for year 2002, and the lowest for year 2005, and similar values are measured for the other years, in agreement with results obtained for $d 1$. For smaller magnetic regions, again the maximum is observed in 2002, but a minimum is found for year 2004 data. We notice that, while suggestive, the trends at larger 


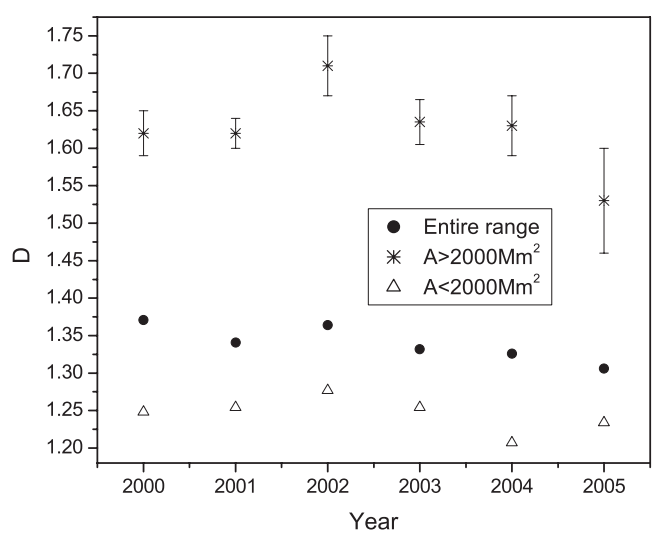

Fig. 4. Temporal variation of the fractal dimension $D$ versus area for selected OAR-PSPT calcium images and for different area range. Error bars in the case of fits performed on the whole dataset (circles) or at smallest objects (triangles) are smaller then the symbol size. Results obtained for the largest area are in good agreement with results obtained by $d 1$ estimator (Fig. 3).

objects are small and the fractal dimension is constant within the measurement uncertainties. Moreover variations of coefficients evaluated over the entire area range and at smaller areas probably reflect variations in the size distribution of the magnetic regions (Meunier 2003; Ermolli et al. 2006) rather than a real temporal variation of the fractal dimension.

\section{Comparison to previous results}

\subsection{Fractal dimension and structure size}

Since the work of Roudier \& Muller (1987) the fractal geometry of structures found in images of the outer layers of the solar atmosphere has been investigated by a number of authors. Both magnetic features, at moderate to high spatial resolution, and non-magnetic features associated with plasma motions have been studied (Roudier \& Muller 1987; Lawrence et al. 1993; Balke et al. 1993; Nesme-Ribes et al. 1996; Berrilli et al. 1998; Meunier 1999; Stenflo \& Holzreuter 2003; Janssen et al. 2003; Meunier 2004; McAteer et al. 2005). In order to ensure a meaningful comparison, we will compare the results we have found here only to those previously published results for active regions investigated using the perimeter-area estimator.

In agreement with previous results, we find a fractal dimension that increases with feature size, from a minimum value of about 1.2 , to an approximately constant value of $1.5-1.7$ for structure areas larger than $\sim 1000-2000 \mathrm{Mm}^{2}$. This range in $d 1$ agrees well with measurements by Nesme-Ribes et al. (1996), but not with those reported by Meunier $(1999,2004)$, who found generally a higher minimum value (around 1.4). From the analysis of both real and simulated data (Sect. 5) we know that the minimum value measured is somewhat dependent on both the identification method employed and the image resolution. We suggest that the higher minimum value reported by Meunier $(1999,2004)$ may be a consequence of image resolution (see Sect. 5.3), as the full-disk MDI data she analyzed are unaffected by atmospheric degradation. The plateau in $d 1$ beyond object sizes of $2000 \mathrm{Mm}^{2}$ also agrees with previous results, but this time more so with those of Meunier (2004) and less so with Nesme-Ribes et al. (1996), who found the plateau to occur already for structures of size $>300$ square-pixels (corresponding to about $500 \mathrm{Mm}^{2}$ ). Tests with both real and simulated data suggest that this difference may lie in the area range over which the fit for $d 1$ at each point was made, a value not quoted by NesmeRibes et al. (1996), but taken in our study to be $\Delta \log A=1.5$ in agreement with that used by Meunier (2004).

An increase of fractal dimension with magnetic feature size was also observed by Janssen et al. (2003). They studied fractal dimension of magnetic features analyzing high resolution (approximately $0.4^{\prime \prime}$ ) magnetograms acquired with the Vacuum Tower Telescope and synthetic images obtained through MHD simulations. They found $D=1.38 \pm 0.07$ on synthetic data, and $D=1.21 \pm 0.05$ on real data, corrected to $D=1.41 \pm 0.05$ when taking in to account resolution effects. Note that our estimate, $D \cong 1.3$ (fit on entire area range), lies in between these last two values. The plots of Janssen et al. (2003) show a deviation from these fits for $\log \left(A / \mathrm{pixel}^{2}\right)>2.5$ (corresponding to $315 \mathrm{pixel}^{2}$ ) for both real and simulated data, despite the differing pixel scale of the two data sets $(\sim 72 \mathrm{~km}$ for real data and $\sim 21 \mathrm{~km}$ for simulated ones). Fits to objects whose areas were larger then this threshold gave $D=1.47$ and $D=1.9$ (values not corrected for resolution effects) for real and simulated objects respectively.

\subsection{Temporal variation}

Meunier (2004) performed a time-dependent analysis, evaluating the variation in the fractal dimension $d 1$ with object size for three different periods: minimum, ascending and maximum phase of the current solar cycle. A correlation with solar activity for structures of size $\sim 1000 \mathrm{Mm}^{2}$ was reported, with the highest fractal dimension being measured during the cycle maximum period. Larger structures $\left(2000-7000 \mathrm{Mm}^{2}\right)$ were found to have a higher fractal dimension during the ascending phase of the cycle than at cycle maximum. Variations were of the order of few per cent. The same trends were found for estimates of $D$, but with larger amplitude variations. If we restrict our analyses to the area range $2000-7000 \mathrm{Mm}^{2}$, we instead find a little correlation of $d 1$ with solar cycle, the highest values being measured for years 2000 and 2002, and the smallest for 2005. The amplitude of the variations in our data is slightly higher than the one reported by Meunier (2004), the largest yearly variation measured over the six year period being of order $10 \%$. The trend reported for structures of moderate size $\left(1000 \mathrm{Mm}^{2}\right)$ is not observed in our analysis.

\section{Discussion of fractal dimension estimation}

Assessment of the fractal dimension of features in digitalized images requires a series of operations:

$$
\begin{aligned}
& \text { - image segmentation to isolate regions of interest; } \\
& \text { - edge identification in the resulting bi-level images; } \\
& \text { - perimeter and area measurement of structures so identified; } \\
& \text { - fractal dimension evaluation using these measures. }
\end{aligned}
$$

Each of these steps introduces a certain degree of arbitrariness which influences the result. Moreover, the results are sensitive to intrinsic differences between image sets, unrelated to the geometric properties of the features they capture. In this section we focus on the effects of edge identification technique, pixelization, and resolution, by analyzing synthetic images of objects whose fractality is known: non-fractal objects, the von Koch curve, and objects obtained by fractional Brownian motion. Seeing effects are also investigated through the analyses of MLSO PSPT data. 

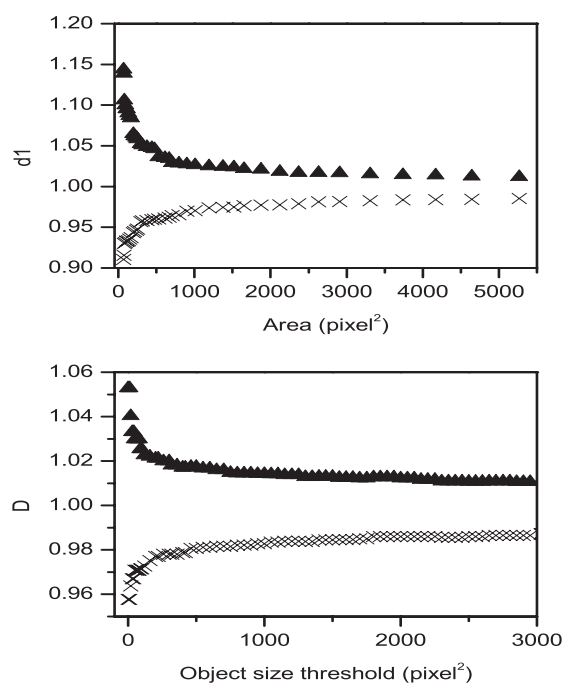

Fig. 5. Fractal dimension $d 1$ (top) and $D$ (bottom) estimated for circles as function of object size and minimum area threshold respectively. Results obtained with external sides (crosses) and 8-contiguous point (triangles) perimeter finding algorithm are shown. Error bars are smaller then symbol size. Note that the horizontal scales differ for the two panels.

\subsection{Perimeter definition and pixelization effects}

To study the influence of the perimeter finding algorithm, we examined the empirical dimension of non-fractal objects as a function of their size. Three different perimeter identification techniques (described in Sect. 2.4) were applied to three geometric shapes (squares, right triangles, and circles). In the absence of error, all three methods should yield a value of one since the objects are non-fractal, but because of image pixelization, fractal dimensions greater or lower than one were measured.

We found that errors in fractal dimension evaluation are functions of the object size for both $D$ and $d 1$. Figure 5 shows the results obtained for circular objects, with the top panel showing $d 1$ versus object area and the bottom panel plotting $D$, evaluated by fitting points of area greater then a given threshold, as a function of the threshold value itself. In both cases, errors are greatest for objects of small size but persist to surprisingly large scales. Analogous trends were observed for the other shapes analyzed. In $d 1$ estimations, errors of less than 5\% are achievable for object sizes greater than some hundreds $-1000 \mathrm{pixel}^{2}$, but for circular objects, which can not be grid aligned, the error never drops below $1 \%$, independent of the perimeter measure employed. This is true even for object sizes exceeding $5000 \mathrm{pixel}^{2}$. For any given size object $D$ is significantly closer to its expected value of 1 than is $d 1$. This is because the evaluation of $D$ in the perimeter-area fit is performed over all points above a minimum size. This includes the large objects not included at small scales in the evaluation of $d 1$. Therefore, the object size threshold above which the error in $D$ is below $5 \%$ occurs at smaller scales than for $d 1$, but still is not usually less than some hundred square pixels.

The origin of these errors lies in the impossibility of representing curves or non-grid aligned lines on a rectangular grid. This causes the area and the perimeter to scale differently from what is expected for non fractal objects. For instance, in the case of a right triangle whose two sides are grid aligned, the overestimation of the hypotenuse leads to the overestimation of both perimeter and area. It can be shown that, because the relative error in the perimeter estimation is not size dependent, while the

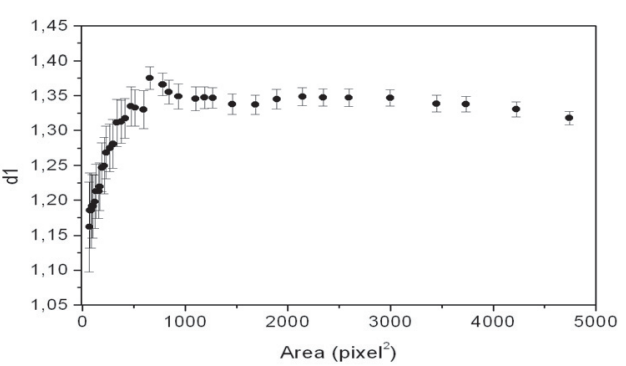

Fig. 6. $d 1$ evaluated for von Koch snowflakes of level 6. Like non fractal objects and real data, $d 1$ increases with object size and reaches a plateau at areas $\geq 1000 \mathrm{pixel}^{2}$. The plateau value, about 1.34 , is an overestimate of the snowflake fractal dimension (see text).

Table 2. Theoretical fractal dimension $D_{\text {th }}$, measured fractal dimension $D$ for the studied objects and the relative error $\epsilon=\left(D_{\text {th }}-D\right) / D_{\text {th }}$. Pixelization errors increase with increasing structure complexity.

\begin{tabular}{cccc}
\hline \hline & $D_{\text {th }}$ & $D$ & $\epsilon$ \\
\hline $\mathrm{fBm}$ & 1.8 & 1.516 & $16 \%$ \\
$\mathrm{fBm}$ & 1.6 & 1.413 & $12 \%$ \\
vonKnoch & 1.26 & 1.310 & $-3 \%$ \\
\hline
\end{tabular}

relative error in area estimation decreases with increasing object size, the estimated fractal dimension is always overestimated.

For our analysis of solar data, we employed only the row and column counting method (the first method described in Sect. 2.4). This method was chosen because it alone produced no error for grid aligned squares, a minimum criterion.

The analysis described above was also applied to fractal structures: the von Koch snowflakes (Peitgen \& Jürgen 1992) and fractional Browian motion (fBm) images (Turner et al. 1998). For the first object, whose fractal dimension is $\sim 1.26$, we produced snowflakes up to level 6 of different sizes (see Appendix) and studied their perimeter and area scaling. For fBms we created two sets of 150 images of expected fractal dimensions 1.8 and 1.6 respectively. Each fBm image was segmented with seven different thresholds (Turner et al. 1998) and perimeter and area of the structures selected by the different thresholds were combined to study the fractal dimension.

In Fig. 6, the measured dimension $d 1$ is plotted as a function of object size for von Koch snowflakes of level 6 . The fractal dimension increases with object size from a minimum value, in this case 1.15 , to an almost constant value approximating the theoretical one, over the size range 1000 to $5000 \mathrm{pixel}^{2}$. The plot is surprisingly reminiscent of that found for real solar structures (Sect. 3). Note that the plateau value, about 1.34, exceeds the one expected theoretically. This reflects the overestimation of the snowflake perimeter inherent in the perimeter measure algorithm employed, as discussed previously for simple non fractal triangle. A rise of fractal dimension with object size was also observed for fBm images. Measurements of $D$ are similarly affected by pixelization at small scales, with more complex structures harder to resolve and thus showing greater measurement error in the deduced fractal dimension, as shown in Table 2.

Both regular and fractal objects show similar pixelization induced errors in the fractal dimension estimation. These effects are greater at smaller areas, where the lack of resolution causes the objects to appear round, thus both $d 1$ and $D$ increase rapidly with object size for object areas less than $\sim 500-1000 \mathrm{pixel}^{2}$ and some hundred pixels square respectively. For objects of larger area, $D$ and $d 1$ increase more slowly, but show deviations from 
Table 3. Fractal dimension measure for different fractals, before $D$ and after $D_{\text {sm }}$ smoothing by convolution with a Gaussian of $F W H M=2$, and the relative error $\epsilon=\left(D-D_{\text {sm }}\right) / D$. Note that to distinguish resolution from pixelization induced effects, the error is evaluated respect to $D$ and not to $D_{\text {th }}$.

\begin{tabular}{ccccc}
\hline \hline & $D_{\text {th }}$ & $D$ & $D_{\text {sm }}$ & $\epsilon$ \\
\hline $\mathrm{fBm}$ & 1.80 & 1.516 & 1.366 & $9.9 \%$ \\
$\mathrm{fBm}$ & 1.60 & 1.413 & 1.303 & $7.8 \%$ \\
von Koch & 1.26 & 1.310 & 1.273 & $2.8 \%$ \\
\hline
\end{tabular}

the expected theoretical value reflecting how the structures map onto the pixel grid.

We thus suggest that the minimum object size thresholds (about some tens of pixel $^{2}$ ) applied in previous works (e.g. Vogelaar \& Wakker 1994) are insufficiently conservative, with residual pixelization effects significantly influencing the final results even for objects of $\sim 1000 \mathrm{pixel}^{2}$.

\subsection{Resolution and seeing effects}

The fractal dimension estimate of solar features depends on the resolution of the images analyzed. Resolution is determined not only by the detector pixel size (image scale), but also by the aperture of the telescope, any instrumental aberration, and, for ground based instrumentation, the distortion introduced by atmospheric turbulence (seeing). Thus the pixel scale and the resolution are not the same. To evaluate the effects of resolution on the estimation of fractal dimension, we analyzed the scaling of $d 1$ and $D$ with area after convolving von Koch snowflake and fBm images with Gaussian functions of different widths. We obtained, as one might have expected, a decrease in both $d 1$ and $D$ accompanying the smoothing. Table 3 of $D$ (fit over the entire perimeter area range) shows also that the smoothing effects become more important as the structure complexity increases.

A Gaussian function is a rough approximation to the seeing and instrumental aberration Point Spread Function in real images. Moreover, seeing is a time dependent phenomenon, so that images acquired at different times are affected by different degradation. In order to investigate directly the effect of variable seeing on the computed fractal dimension of structures in real data, we examined full resolution PSPT images from MLSO after selection based on the quality criteria described in Sect. 2.2. Images were segmented with the first technique explained in Sect. 2.3. Figure 7 shows that a real decrease in resolution resulting from degraded observing conditions leads to an underestimation of features' complexity at all scales.

\section{Interpretation of results measured}

We have shown that the determination of fractal dimension of features in digitalized images by the two estimators $d 1$ and $D$ is affected by pixelization and resolution. Understanding these effects is essential to the interpretation of the results obtained from OAR and MLSO PSPT images (Sect. 3) as well as those previously reported from studies carried out with similar techniques on other data. Pixelization errors occur at all scales, but are generally more important for the smallest objects. This causes the estimated fractal dimension to increase rapidly with object size and become almost constant at areas larger than a critical threshold, that we estimated to be $\sim 500-1000$ pixel $^{2}$ for $d 1$ and some hundreds of pixel $^{2}$ for $D$. Seeing and instrumental induced image degradation smooths edges making structures appear rounder,

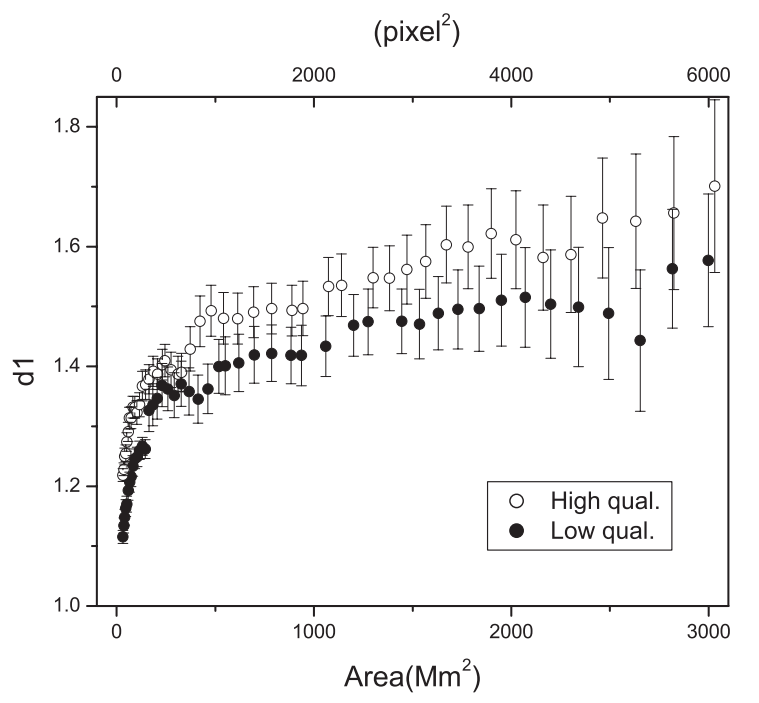

Fig. 7. Facular fractal dimension estimated on the two different full resolution MLSO quality sets described in the text. When the estimation is carried out on images less affected by seeing degradation, the measured fractal dimension is higher.

resulting in a reduced fractal dimension. This effect is expected, on the basis of synthetic fractal data, to be more important for more complex objects (Sect. 5.2).

We thus suggest that the rise of $d 1$ with object size observed in PSPT data, as well as for example in Meudon spectroheliograms (Nesme-Ribes et al. 1996) and MDI magnetograms (Meunier 1999, 2004), is most likely an effect of image pixelization, rather than a signature of an intrinsic multifractality of active regions. Conclusions drawn in Meunier (2004) concerning a change in physical properties of magnetic structures at supergranular scales, should thus be reviewed in light of the results shown in this paper. Pixelization is also likely the cause of the "break of similarity" observed by Janssen et al. (2003), the break occurring at the same pixel scale for both real and simulated images, in spite of the different physical scale implied, and in the same area range suggested by our synthetic fractal studies.

For larger objects, the fractal dimension estimate is most affected by seeing. The values measured for large scale structures in the PSPT observations (1.5 to 1.7) are therefore likely an underestimate of the real value. Nevertheless, in some cases pixelization can cause overestimation of the fractal dimension at largest areas, as shown for instance for the von Koch snowflake. We cannot therefore in principle exclude some compensation due to the combined effect of pixelization and reduction of resolution. We finally note that MDI magnetograms, while not affected by seeing, are slightly defocused, so that the resolution is twice that of the pixel scale (Scherrer et al. 1995). The same considerations made for results obtained with ground based measurements thus also apply to results obtained with MDI.

A study of the fractal geometry of solar active regions and its variation with the magnetic activity cycle is thus feasible if it focuses only on large features, employs a constant segmentation technique throughout, and utilizes data of consistently high quality. The OAR PSPT images analyzed over a period of six years marginally meet these requirements. They do not show, however, a clear correlation with the solar cycle. Moreover the variations measured in D appear to be dominated by variations in size distribution of the examined features (Ermolli et al. 2006), which in turn weight the perimeter-area fit. 


\section{Conclusions}

We have analyzed the fractal dimension of bright features identified in solar chromospheric CaII K images obtained during the last six years, corresponding to the descending phase of solar cycle 23 . The results obtained are in general agreement with those reported in literature, in particular with those studies that have been carried out with similar fractal estimators $(d 1$ and $D)$.

We have also investigated the effects of pixelization and resolution on the fractal dimension estimates obtained, studying these effects on real and simulated data, the latter including both non-fractal objects and objects whose fractal properties are well known, von Koch snowflakes and fBm images. We have shown that fractal dimension estimates suffer from pixelization errors at all scales, but errors are generally more important for areas less than 500-1000 pixel $^{2}$. Particularly, pixelization causes measured fractal dimensions to increase with object size in the case of both fractal and non-fractal objects. Our results thus indicate that the increase of fractal dimension with the feature size reported in literature by some previous analyses is likely an effect of pixelization and image degradation, rather than a signature of an intrinsic multifractality of active regions. To reduce these effects, we thus suggest a restriction of the analyses to objects whose areas are larger then the quoted value.

Our analyses also showed that image degradation due to both seeing and instrumental effects smooths features edges making them appear rounder. The fractal dimension estimated is consequently lower than expected, even for large objects. Perhaps image degradation effects can be compensated for, as suggested in Janssen et al. (2003). Alternatively, a more careful estimate of these effects can be carried out by the analyses of images of known complexity objects (e.g. fBms) convolved with realistic Point Spread Functions. We did not apply either of these compensations to our data and leave them for future investigation.

Finally we note that, as demonstrated by Baveye et al. (1998) for the box counting method, pixelization effects can influence other fractal dimension estimators as well, and careful quantitative measure of the effects for each measure employed is essential to the interpretation of the results. We have not addressed this problem in this work and leave also this issue for future research.

Acknowledgements. The authors acknowledge useful comments by J. K. Lawrence that refereed the article. The authors are also grateful to D. Del Moro, G. Consolini and H. Liu for fruitful discussions.

\section{References}

Balke, A. C., Schrijver, C. J., Zwaan, C., \& Tarbell, T. D. 1993, Sol. Phys., 143, 215

Baveye, P., Boast, C. W., Ogawa, S., Parlange, J., \& Steenhuis, T. 1998, Water Resources Research, 34, 2783

Berrilli, F., Florio, A., \& Ermolli, I. 1998, Sol. Phys., 180, 29

Ermolli, I., Criscuoli, S., Centrone, M., \& Giorgi, F. 2006, in press

Gonzalez, R. C., \& Woods, P. E. 2002, Digital Image processing (London: Prentice Hall)

Harvey, K. L., \& White, O. R. 1999, ApJ, 515, 812

Janssen, K., Vögler, A., \& Kneer, F. 2003, A\&A, 409, 1127

Lawrence, J. K., Ruzmaikin, A. A., \& Cadavid, A. C. 1993, ApJ, 417, 805

Lawrence, J. K., Cadavid, A. C., \& Ruzmaikin, A. A. 1996, ApJ, 465, 425

McAteer, R. T. J., Gallagher, P. T., \& Ireland, J. 2005, ApJ, 631, 628

Meunier, N. 1999, ApJ, 515, 801

Meunier, N. 2003, A\&A, 405, 1107

Meunier, N. 2004, A\&A, 420, 333

Nesme-Ribes, E., Meunier, N., \& Collin, B. 1996, A\&A, 308, 2213

Ortiz, A., \& Rast, M. 2005, Mem SAIt., 76, 4, 1018

Peitgen, H. O., \& Jürgen, D. 1992, Fractals for the classroom, Part One, Introduction to Fractals and Chaos (New-York: Springer-Verlag)

Rast, M. P. 2003, Local and Global Helioseismology: The Present and Future, Proceedings of SOHO12/GONG+ 2002, ed. H. Sawaya-Lacoste, ESA SP517,163

Roudier, Th., \& Muller, R. 1987, Sol. Phys., 107, 11

Sànchez, N., Alfaro, E. J., \& Pèrez, E. 2005, ApJ, 625, 849

Scherrer, P. H., et al. 1995, Sol. Phys., 162, 129

Skumanich, A., Smythe, C., \& Frazier, E. N. 1975, ApJ, 200, 747

Stenflo, J. O., \& Holzreuter, R. 2003, ASP Conf. Ser., 286, 169

Turner, M. J., Blackledge, J. M., \& Andrews, P. R. 1998, Fractal Geometry in Digital Imaging (London: Academic Press)

Vogelaar, M. G. R., \& Wakker, B. P. 1994, A\&A, 291, 557 


\section{Online Material}




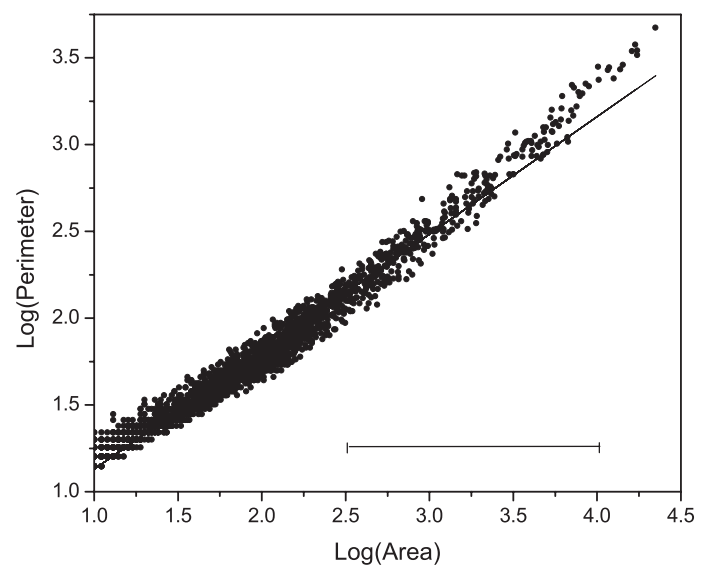

Fig. A.1. Perimeter (in units of pixel) and Area (in units of pixels square) in logarithmic scale of detected structures on OAR PSPT data taken during summer 2002. Continuous line is the fit to the whole set of data $(D=1.35)$. Points at area larger than about 1000 pixels square are better approximated by a higher slope line. Horizontal line is the area window width over which $d 1$ is estimated.

\section{Appendix A: On the estimation of $D$ and $d 1$ on digitalized images}

The estimators adopted in this paper to evaluate fractal dimension of selected structures are based on the perimeter-area relation. This consists of measuring the perimeter and area of a structure at different resolutions. For regular structures, perimeter scales as the square root of the area, while for fractal structures the exponent is greater than 0.5. By definition, the exponent is the fractal dimension of the studied object. Note that in Sect. 2.5 we normalized the exponent so that the fractal dimension is one for regular structures and greater than one for fractal objects. It's worth noticing that, when adopting this method to investigate fractal nature of magnetic solar regions, one implicitly assumes that the different size selected structures are the "same" object observed at different resolution.

When plotting the perimeter-area relation of real data, one expects to find at least three different regimes. At the smallest sizes, because of resolution, an object's detail is is not fully detected, thus perimeter and area scale as for regular non-fractal structures. At the largest areas, a break in similarity can occur for physical reasons (for instance the object under study has a finite maximum size above which it is no longer a fractal). At intermediate areas the object scales as a fractal. As an example, in Fig. A.1 we plot the perimeter-area relation for structures selected in OAR-PSPT images. The straight line is a linear fit to the points. It's evident that points don't lie on a single line, but rather on a curve. One is thus tempted to measure the tangent to the curve as a "local" measure of the fractal dimension. This is what the measure in $d 1$ employed in the text does. All our plots (cf. Figs. 2, 3, 5-7) showed that $d 1$ increases with object size, becoming eventually constant at some typical scale (generally in the area range 500-1000 square pixels). The measured $d 1$, as well as the area at which it becomes constant, are functions of the window size. Nevertheless, when using this estimator, it is important to keep in mind that any fractal estimate requires the autosimilarity to be valid over some orders of magnitude (Baveye et al. 1998) (the area range $\Delta \log A=1.5$ adopted for $d 1$ in this and in other works is therefore in principle too small).

The results we obtained with von Koch snowflakes illustrate clearly these issues. von Koch snowflake images of different sizes were produced following the iterative scheme of
Peitgen \& Jürgen (1992). After each iteration, or level, the snowflake is more structured, with an increase in both perimeter and area. In the limit of infinite iterations, the perimeter tends toward infinity and the area approaches a finite value. Here we investigate structures constructed with up to 6 levels. The fractal dimension of the von Koch snowflake is $\log 4 / \log 3 \approx 1.26$.

In left panel of Fig. A.2, the perimeter-area relationships for snowflakes of levels 2,4 , and 6 are plotted with logarithmic scaling. For each level, the relationship traces a curve made up of segments whose slope is $1 / 2$ connected by segments of slope greater than $1 / 2$. At largest areas all the points lay on parallel lines of slope $1 / 2$. At those scales the snowflakes of all the represented levels are fully resolved on the grid employed. As the dimensions of the objects are reduced, fewer details at any fixed construction level are resolved, the measured perimeter decreases at a rate faster than $A^{1 / 2}$, and the perimeter-area curve steepens. The slope flattens to a value of one-half again each time the grid resolution is sufficient to capture the details of the next lower level. Finally, at smallest areas most geometric details are lost and all the objects, independent of their initial construction level, appear non-fractal.

The scaling of $d 1$ better reflects the change in slope with objects size. As an example, in right panel of Fig. A.2 we show results obtained for level 6 . Here full and open dots represent respectively $d 1$ obtained with a window of $\Delta \log A=1.5$ and a window of $\Delta \log A=0.5$. With the largest window only the slope change that occurs at largest areas is visible. The others occur on scales smaller than the window so that they are not "detected" and a plateau is observed. At smallest areas $d 1$ drops because of the resolution effects explained before. When a smaller window is used, 6 peaks are visible, corresponding to the 6 slope-changes visible in the perimeter area scatter plot. In this case, there is an area range over which fractal dimension oscillates around a constant value. At smallest areas larger amplitude oscillations are observed. Both curves show clearly two of the three regimes mentioned above. The object scales as a fractal in the range $3<\log ($ Area $)<4.5$. At smaller areas, pixelization effects dominate the measurements because resolution is insufficient to allow detection of all the structure's details. The third regime is not evident in the two curves, since not enough points are available at largest areas to perform the fit. If more points were available we would observe a decrease of $d 1$ toward the value of one, as slightly visible at largest areas for fits performed on the smaller window. 
S. Criscuoli et al.: Solar magnetic features fractal dimension: reliability and variation with solar cycle, Online Material p 3
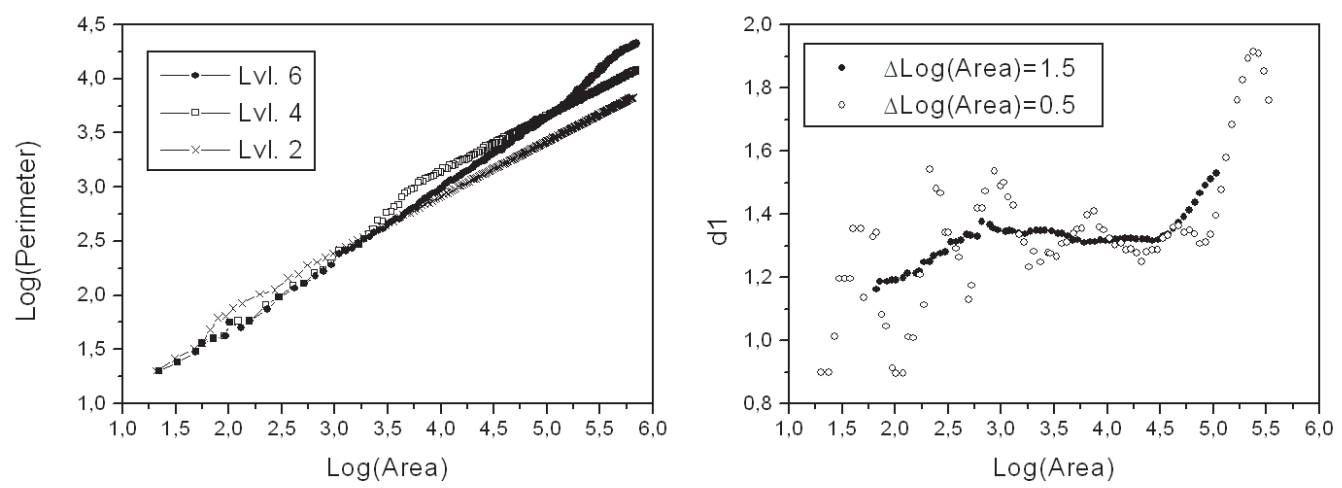

Fig. A.2. Left: perimeter versus area in logarithmic scale of snowflakes of levels 2, 4, and 6. Because of pixelization, these structures exhibit a fractal scaling only within certain area ranges, the bounds depending on the snowflake level. Right: $d 1$ versus area evaluated with different window sizes for snowflakes of level 6. Peaks obtained with the small window (open circles) are due to the steep variations visible in plot on the left. Peaks are not detected with a larger window (full circles). The area range over which $d 1$ is almost constant is the range over which the simulated images are fractal. 